\title{
Comparison of Anti-Microbic and Anti-Biofilm Activity Among Tedizolid and Radezolid Against Linezolid-Resistant Enterococcus faecalis Isolates
}

\author{
Lingbo Wang ${ }^{1} *$ \\ Ying Zhang ${ }^{2, *}$ \\ Shixing Liu' \\ Na Huang' \\ Weiliang Zeng' \\ Wenya Xu' \\ Tieli Zhou' \\ Mo Shen'
}

'Department of Clinical Laboratory, The First Affiliated Hospital of Wenzhou Medical University, Wenzhou, Zhejiang Province, People's Republic of China; ${ }^{2}$ School of Laboratory Medicine and Life Science, Wenzhou Medical University, Wenzhou, Zhejiang Province, People's

Republic of China

*These authors contributed equally to this work
Correspondence: Mo Shen; Tieli Zhou Department of Clinical Laboratory, The First Affiliated Hospital of Wenzhou Medical University, Wenzhou, People's Republic of China

Tel +86-577-8668-9885

Email shenmo601@I63.com;

wyztli@I63.com
Background: The emergence and spread of linezolid-resistant Enterococcus faecalis (E. faecalis) have emerged as a serious threat to human health globally. Therefore, this study aims to compare the anti-microbic as well as the anti-biofilm activity of linezolid, tedizolid, and radezolid against linezolid-resistant E. faecalis.

Methods: A total of 2128 E. faecalis isolates were assessed from the First Affiliated Hospital of Wenzhou Medical University from 2011 to 2019. Antibiotic sensitivity was evaluated using the micro broth dilution method. Oxazolidinone-resistant chromosomal and plasmid-borne genes such as $c f r, c f r(A), c f r(B), c f r(C), c f r(D)$, optrA, and poxtA were detected by PCR and then sequenced to detect the presence of mutations in the domain $\mathrm{V}$ of the $23 \mathrm{~S}$ rRNA and the ribosomal proteins L3, L4, and L22. Conjugation experiments were conducted using the broth method. The inhibition and eradication of biofilm were evaluated through crystal violet staining, whereas the efflux pump activities were detected by agar dilution.

Results: Out of 2128 isolated E. faecalis, 71 (3.34\%) were linezolid-resistant isolates in which the MICs of tedizolid and radezolid ranged from 1 to $4 \mu \mathrm{g} / \mathrm{mL}$ and $0.5-1 \mu \mathrm{g} / \mathrm{mL}$, respectively. The $\mathrm{MIC}_{50} / \mathrm{MIC}_{90}$ of tedizolid and radezolid were 4 and 8 -fold lower than the linezolid, respectively. Out of 71 resistant isolates, 57 (80.28\%) carried optrA, 1 (1.41\%) carried $c f r, 4(5.63 \%)$ carried optrA and $c f r$, and $6(8.45 \%)$ carried optrA and $c f r(D)$, with no mutations of 23S rRNA gene and ribosomal proteins L3, L4, and L22. Besides, the transfer rate of the optrA, cfr, and $c f r(D)$ was $17.91 \%, 0 \%$ and $0 \%$, respectively. Radezolid showed more effectiveness in eradicating biofilm $(8 \times$ MIC). However, tedizolid was more effective than radezolid and linezolid in inhibiting the biofilm formation (1/4 MIC, 1/8MIC, and 1/16MIC). Additionally, in combination with CCCP, the MICs of radezolid in all linezolid-resistant isolates decreased $\geq 4$-fold.

Conclusion: Radezolid showed greater antimicrobial activity than tedizolid and linezolid against linezolid-resistant $E$. faecalis. However, both tedizolid and radezolid showed differential activity on biofilm inhibition, eradication, and efflux pump compared to linezolid. Thus, our study might bring important clinical value in the application of these drugs for resistant pathogenic strains.

Keywords: Enterococcus faecalis, linezolid, tedizolid, radezolid, in vitro activity, antibiofilm activity

\section{Introduction}

Enterococcus is a Gram-positive bacteria organism that is the most common hospital-acquired infection. ${ }^{1}$ It is a significant public concern globally due to substantial morbidity and mortality worldwide. ${ }^{2}$ Enterococcus faecalis (E. faecalis) is a pathogenic strain and accounts for approximately $97 \%$ of all 
infective endocarditis (IE) cases, as a hospital acquired infection. $^{3}$ In recent years, multidrug-resistant (MDR) E. faecalis has spread rapidly with its high ability to form biofilm. Unfortunately, the MDR infections caused by $E$. faecalis are hard to cure, making the clinical treatment ever challenging. Besides, the choices for antiinfective therapies are further narrowed due to the intrinsic and acquired resistance of E. faecalis. ${ }^{4}$ The limited availability of novel antimicrobial agents is also posing difficulties for anti-infection treatment in clinics. Thus, new effective ways for the treatment of MDR E. faecalis infection are urgently warranted.

Oxazolidinone is a new class of antibacterial agents that inhibits bacterial protein synthesis by preventing the binding of the aminoacyl-tRNA to the site of the ribosome. ${ }^{5-7}$ Linezolid was the first clinically helpful oxazolidinone antibacterial agent approved by the US Food and Drug Administration (FDA). ${ }^{8}$ However, linezolidresistant Staphylococcus aureus and Enterococcus spp emergence have risen in recent years due to the extensive use of linezolid in clinical practice. ${ }^{9}$ Therefore, it is extremely important to develop new oxazolidinone antimicrobial agents.

Tedizolid, a novel oxazolidinone prodrug, is the second-generation oxazolidinone approved by the FDA (Food and Drug Administration). It exerts its antibacterial activity by binding to the peptidyl transferase center (PTC) of the 50S subunit of the bacterial ribosome, making it more active against enterococci than linezolid. ${ }^{10}$ Moreover, structural differences between the C- and D-loop creates additional target site for interaction with 23S rRNA residues to form the PTC binding site, enhancing the effectiveness of tedizolid. ${ }^{11}$ Radezolid is another oxazolidinone antimicrobial agent possessing enhanced antibacterial potency against antibiotic-resistant Grampositive bacteria compared with linezolid. It exerts its antimicrobial activity by forming $\pi-\pi$ stacking interactions with the 50S subunit of the bacteria. ${ }^{12}$ Till now, only a few studies have compared the antibacterial activity of tedizolid and radezolid with linezolid against the Staphylococcus aureus and Enterococcus. ${ }^{13,14}$ However, the comparison of in vitro activity of these two oxazolidinone agents against linezolid-resistant $E$. faecalis remains unknown.

Linezolid resistance is unusual among enterococci, however, the number of linezolid-resistant strains has increased gradually over the years. The resistance in enterococci is generally contributed by the point mutations in the chromosomal 23S rRNA gene, although the amino acid change G2576T being the predominant one, other changes (G2505A, U2500A, G2447U, C2534U, and $\mathrm{G} 2603 \mathrm{U}$ ) have also been reported; ${ }^{15}$ Mutations in the ribosomal proteins L3, L4, and L22 encoded by rplC, rplD, and rplV genes contributed to linezolid resistance. ${ }^{16}$ On the other hand, the acquisition of the plasmid-borne genes $(c f r, \quad c f r(B), \quad c f r(D), \quad o p t r A$, and poxtA) can also cause resistance from linezolid. ${ }^{17,18}$ The cfr gene and its variants $\operatorname{cfr}(\mathrm{B})$ and $\operatorname{cfr}(\mathrm{D})$ have also been reported in the resistance to oxazolidinones. This encodes for rRNA methyltransferase, which modifies the adenine residue at the 2503 position of domain $\mathrm{V}$ in the $23 \mathrm{~S}$ rRNA. ${ }^{19}$ The optrA gene encodes the ATP-binding cassette $\mathrm{F}$ (ABC-F) protein and mediates resistance to oxazolidinones by protecting the bacterial ribosome. PoxtA is another gene associated with decreased susceptibility to oxazolidinones. ${ }^{17}$ Tedizolid possesses greater in-vitro potency than its first-class predecessor, linezolid. The G2576T mutation ${ }^{20}$ are known to generate resistance against tedizolid, and the presence of optrA ${ }^{21}$ are known to generate resistance against tedizolid. Although fewer studies report the resistance to radezolid, it is related to the mutations in the $\mathrm{V}$ domain of $23 \mathrm{~S}$ rRNA. ${ }^{22}$ Biofilms are enclosed exopolymer matrix on bacteria, restricting the diffusion as well as the penetration of antimicrobials. Although biofilms structure reduces the antibacterial effect, linezolid has been reported to have good inhibitory effects on E. faecalis biofilms, ${ }^{23,24}$ which may be related to the time and dosage of treatment and the inhibition of the number of bacteria in the growing biofilm. ${ }^{25}$ Besides, previous research has reported that the MICs for linezolid or radezolid showed a 2 to 8 fold decreased by the presence of PA $\beta \mathrm{N}$ (efflux pump inhibitors). ${ }^{26}$ Although tedizolid and radezolid belong to the same oxazolidinones class drugs, the differences in their antimicrobial activity against linezolid-resistant E. faecalis are still unclear. Moreover, the variable effect of tedizolid and radezolid on the biofilm of linezolid-resistant E. faecalis compared with linezolid and the role and differences of efflux pump in these three generations oxazolidinones resistance is still largely unknown.

Therefore, the current study was carried out to understand and compare the antimicrobial as well as the antibiofilm activity among linezolid, tedizolid, and radezolid against linezolid-resistant clinical E. faecalis. Further, the differences in the effect of biofilm and efflux pump inhibitors among three antimicrobial agents were also assessed. The result of this study is expected to make an 
essential contribution to the control of hospital-acquired infections caused by the epidemic of E. faecalis.

\section{Materials and Methods}

\section{Bacterial Strains and Antimicrobial Susceptibility Testing}

During the year 2011-2019 period, 2128 clinical E. faecalis isolates were collected from the First Affiliated Hospital of Wenzhou Medical University, China. Identification of the isolates were performed by the matrix-assisted laser desorption/ionization-time of flight mass spectrometry (MALDITOF MS; bioMérieux, Lyons, France). In addition, duplicate strains isolated from the same part of the same patient were removed during strains collection. Minimum inhibitory concentrations (MICs) of linezolid, tedizolid and radezolid were determined by the microdilution broth method with cationadjusted Mueller-Hinton broth based on the Clinical and Laboratory Standards Institute (CLSI, 2020), ${ }^{27}$ whereas the E. faecalis ATCC 29212 was used for the quality control strain.

\section{Detection of Linezolid Resistance Genes}

The DNA of linezolid-resistant $E$. faecalis isolates were extracted using a Bioflux bacterial DNA Extraction Kit. The 23S rRNA and ribosomal proteins L3, L4, and L22 were amplified by the polymerase chain reaction (PCR) using the specific primers ${ }^{28}$ (Table S1). The amplified PCR products were sequenced by Shanghai Majorbio Bio-Pharm Technology Co. (Shanghai, China). Further, the sequences were aligned to analyze any possible gene mutations by BLAST on the National Center for Biotechnology Information (NCBI) (http://blast.ncbi.nlm.nih.gov/Blast. cgi). The transferable resistance genes $c f r, c f r(A), c f r(B)$, $c f r(C), c f r(D)$, optrA and potxA were also screened by PCR assays using the primers ${ }^{29}$ listed in Table S1.

\section{Conjugation Experiments}

Filter mating was conducted for the $c f r$-, $c f r(D)$ - and optrApositive strains of E. faecalis $\mathrm{JH} 2-2$ as recipients, based on the previously reported method. ${ }^{30}$ Thereafter, the transconjugants were selected on Mueller-Hinton agar plates supplemented with $10 \mu \mathrm{g} / \mathrm{mL}$ florfenicol and $25 \mu \mathrm{g} / \mathrm{mL}$ rifampicin. The transconjugants were further confirmed by PCR.

\section{Biofilm Biomass Assay}

The inhibition and eradication of biofilm by linezolid, tedizolid or, radezolid against the $8 \mathrm{E}$. faecalis clinical isolates
(FC1996, FC2021, FC2234, FC2257, FC2279, FC2309, FC2471, FC2484) were performe. The crystal violet staining detected isolates were randomly selected as previously described. ${ }^{31}$ For biofilm eradication assay, E. faecalis strains were inoculated into 96 -well polystyrene microtiter plates with Luria-Bertani broth $(1 \% \mathrm{NaCl}, 1 \%$ tryptone, $0.5 \%$ yeast extract) for the formation of mature biofilms. After $24 \mathrm{~h}$ of static incubation, the supernatants were discarded, and the plates were washed with $0.9 \%$ saline to remove unattached cells. Thereafter, fresh Luria-Bertani broth containing linezolid, tedizolid or radezolid $(8 \times \mathrm{MIC})$ was added to the wells. Till the $48 \mathrm{~h}$ of static incubation, the medium was replaced daily, and at the end of incubation the remaining biofilm biomass was determined by crystal violet staining. The biofilms biomass formed in 96-well polystyrene microtiter plates was determined by detecting the absorbance at $595 \mathrm{~nm}$. Additionally, the inhibition of E. faecalis biofilm formation by linezolid, tedizolid, or radezolid were also investigated based on the previous study. ${ }^{32}$ Briefly, the E. faecalis isolates were inoculated into 96-well polystyrene microtiter plates with Luria-Bertani broth containing linezolid, tedizolid or radezolid (at 1/4MIC, 1/8MIC, 1/16MIC). After $24 \mathrm{~h}$ of static incubation, the biofilm biomass was determined by the crystal violet staining. Each assay was performed in triplicate for at least three times.

\section{Effect of Efflux Pump Inhibitor}

The efflux pump activities in linezolid-resistant isolates were detected by using the efflux pump inhibitor carbonyl cyanide m-chlorophenylhydrazone (CCCP). The MICs for linezolid, tedizolid, and radezolid were determined in the presence or absence of the CCCP $(10 \mu \mathrm{g} / \mathrm{mL})$. Wherever the MIC of the strain decreased $\geq 4$-fold after supplementing efflux pump inhibitor, the phenotype test regarded as positive.

\section{RT-qPCR to Determine the Expressive Levels of $E$. faecalis Efflux Pump-Related Genes}

The expression levels of E. faecalis (FC2234 FC2559 FC1728 FC1037 FC2398 FC2471) efflux pump-related genes (OG1RF12220, OG1RF10126, and OG1RF10495) were determined by RT-qPCR based on published reports. ${ }^{26}$ Real-time qPCR was performed using the 7500 RTPCR System (Thermo Fisher Scientific, Marsiling, Singapore) equipped with the SYBRTM Green RT-PCR Kit (TOYOBO, Osaka, Japan). The primers used for qRT-PCR are listed in Table S1. The 
strains were inoculated on a blood plate at $37^{\circ} \mathrm{C}$ for $16-$ $18 \mathrm{~h}$. A single colony was selected and cultured in LB medium with shaking at $180 \mathrm{rpm}$ until reaching the logarithmic phase at $37^{\circ} \mathrm{C}$. The bacterial suspension was then harvested for RNA extraction by using the Trizol method. The PCR reaction was mixed well and microcentrifuged. Then, $10 \mu \mathrm{L}$ of the mixture was transferred into the qPCR reaction plate, followed by qRTPCR, as suggested by the manufacturer. The $16 \mathrm{~S}$ rRNA gene was simultaneously used as a reference gene for data normalization, and the expression of each gene was determined by the $2^{-\Delta \Delta} \mathrm{Ct}$ method. All experiments were performed in triplicate and repeated thrice independently.

\section{Results}

\section{Antimicrobial Susceptibility Testing}

Among the 2128 E. faecalis collected, 3.34\% (71/2128) of E. faecalis isolates were resistant to the linezolid. Table 1 and Figure 1 show the MICs of linezolid, tedizolid, and radezolid against the linezolid-resistant isolates. Among the 71 linezolid-resistant isolates, the MICs of tedizolid and radezolid ranged from 1 to $4 \mu \mathrm{g} / \mathrm{mL}$, and $0.5-1 \mu \mathrm{g} /$ $\mathrm{mL}$, respectively. The $\mathrm{MIC}_{50} / \mathrm{MIC}_{90}$ of tedizolid and radezolid exhibited 4- and 8-fold lower response than linezolid, respectively. This data suggests that in vitro activity of radezolid against 71 linezolid-resistant isolates was higher than tedizolid and linezolid.

\section{Molecular Mechanisms of \\ Linezolid-Resistant Isolates}

Among the 71 linezolid-resistant E. faecalis clinical isolates, 57 isolates $(80.28 \%)$ carried optrA only, 1 (1.41\%) carried $c f r, 4(5.63 \%)$ carried both optrA and $c f r$, simultaneously, whereas the $6(8.45 \%)$ isolates carried optrA and $c f r(D)$, simultaneously. Interestingly, $c f r(A), c f r(C)$, and $\operatorname{potx} A$ genes were not detected in any of the isolates. Besides, no mutation for linezolid resistance was detected in the domain $\mathrm{V}$ of the 23S rRNA gene nor in the ribosomal proteins L3, L4, and L22. Detailed data of resistance mechanisms are provided in Table 1, Table S2 and Figure 1.

\section{Transferability of Linezolid Resistance}

The optrA and $c f r$ genes were successfully transferred from $17.54 \%(10 / 57)$ optrA-positive isolates and $0 \%(0 /$ 1) $c f r$-positive to $E$. faecalis $\mathrm{JH} 2-2$, respectively. Furthermore, among four isolates carrying optrA and $c f r$

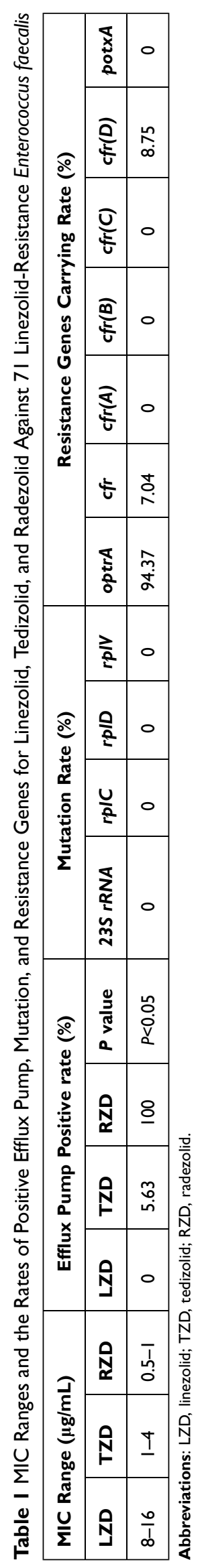




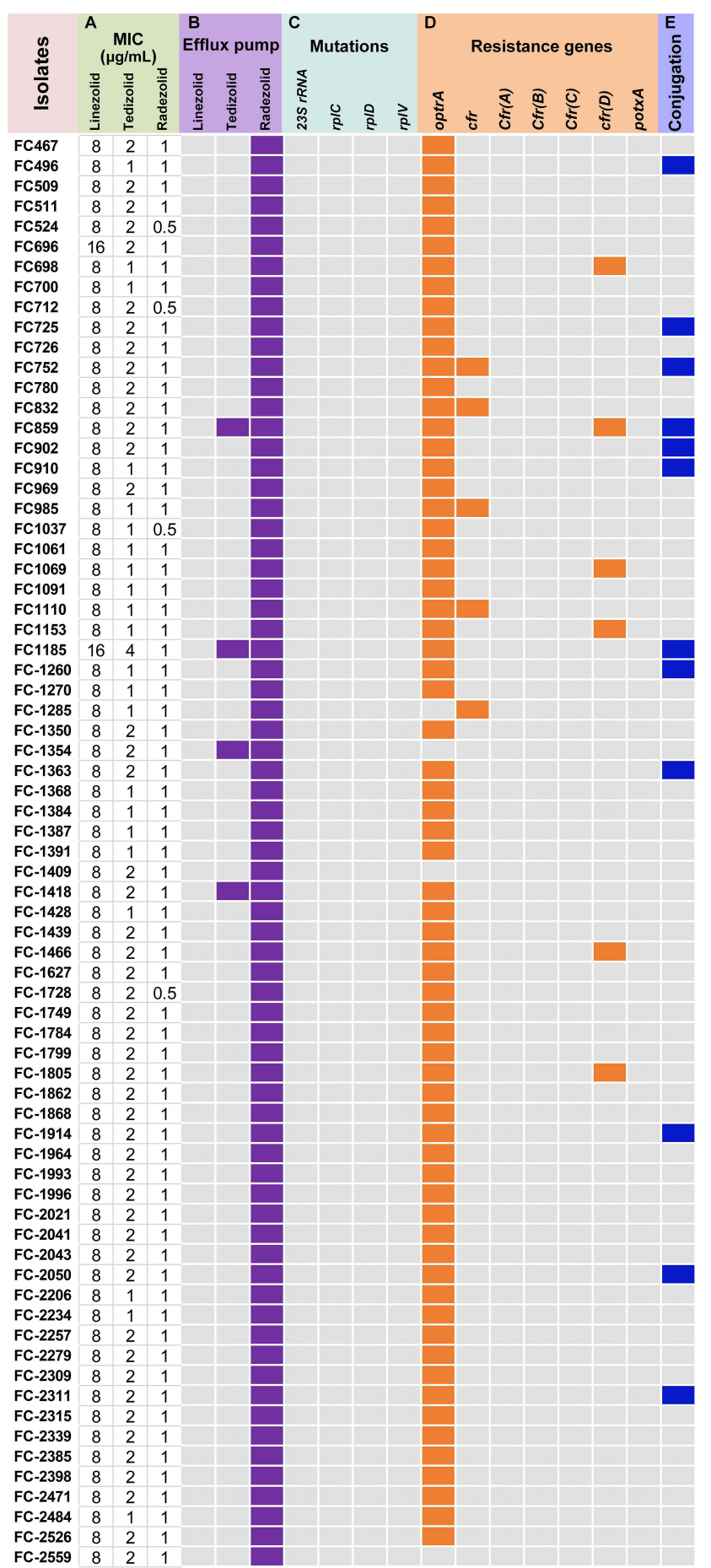

Figure I MIC ranges and the rates of positive efflux pump, mutation, and resistance genes for linezolid, tedizolid, and radezolid against 7/ linezolid-resistance Enterococcus faecalis (A) MICs; (B) carbonyl cyanide 3-chlorophenylhydrazone (CCCP) was used to detect the activity of efflux pumps; (C) the mutation of 235 rRNA and ribosomal proteins L3, L4, and L22; (D) the resistance genes of oxazolidinones; (E) the conjugation of resistance genes of oxazolidinones. Purple, Orange and blue squares represent positive, gray squares represent negative.

genes, one isolate transferred the optrA gene. Similarly, among six isolates carrying optrA and $\operatorname{cfr}(D)$ genes, one isolate successfully transferred the optrA gene. The transfer rate of the optrA, $c f r$, and $c f r(D)$ were $17.91 \%$ $(12 / 67), 0 \%(0 / 5)$ and $0 \%(0 / 6)$, respectively (Figure 1).

\section{Difference of Biofilm Formation Inhibition and Biofilm Clearance Ability of Three Antimicrobial Agents}

The 8 linezolid-resistant $E$. faecalis clinical isolates (FC1996, FC2021, FC2234, FC2257, FC2279, FC2309, FC2471, and FC2484) were randomly selected for comparing the differential effects of linezolid, tedizolid and radezolid on E. faecalis biofilms. First, we compared the eradicating potential of linezolid, tedizolid, and radezolid $(8 \times \mathrm{MICs})$ on established E. faecalis biofilms. In most strains (FC1996, FC2234, FC2257, FC2279, FC2309, and FC2484), radezolid had a better clearance effect on biofilms than with linezolid and tedizolid, however, in 2 strains (FC2021 and FC2471) it was not statistically different. In general, for the biofilm clearance of linezolid-resistant strains of $E$. faecalis, radezolid has the best clearance effect, followed by linezolid, in most strains, tedizolid has a poor clearance effect on biofilm (Figure 2A). We also compared the biofilm formation inhibiting the ability of three antibiotics of oxazolidinone class in E. faecalis at the $1 / 4 \times \mathrm{MIC}, 1 / 8 \times \mathrm{MIC}$, and $1 / 16 \times \mathrm{MIC}$. As shown in Figure 2B-D, most of the three antibacterial agents had a better inhibition effect on the formation of biofilm. Still, the inhibition effect varied as tedizolid at $1 / 4 \times$ or $1 /$ $8 \times$ MIC can efficiently inhibit the biofilm formation of E. faecalis, besides in most isolates, it had greater effect than linezolid and radezolid.id. This similar trend was also observed in several of the eight clinical isolates at MICs of $1 / 16 \times$.

\section{The Potential Effect of the Efflux Pump Mechanism}

The correlation between efflux pump and three different generations of oxazolidinones against linezolid-resistant E. faecalis was tested. The results showed that after being exposed to efflux inhibitor CCCP $(10 \mu \mathrm{g} / \mathrm{mL})$, the MIC values of linezolid was in none of the strains decreased by 4 -fold or more and the MICs of tedizolid decreased 4-fold only on 4 (5.63\%) linezolid-resistant E. faecalis. Moreover, the MICs of radezolid on all linezolid-resistant isolates decreased $\geq 4$-fold in combination with CCCP $(10 \mu \mathrm{g} / \mathrm{mL})$ (Tables 1, S3 and Figure 1). Among the three antimicrobial agents, the MICs of radezolid decreased most significantly, and the results showed that the effluent pump had the most significant 

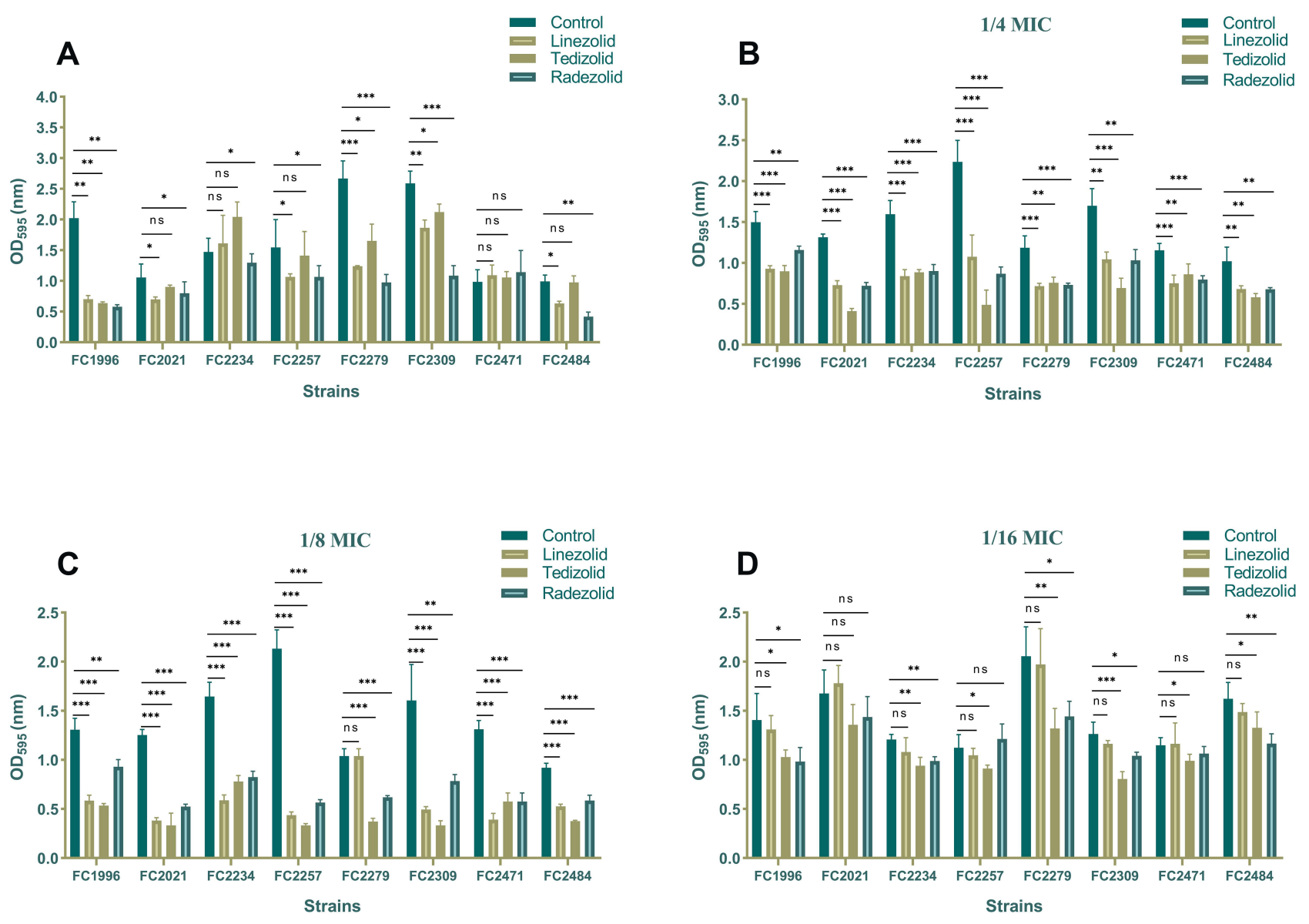

Figure 2 The effect of Linezolid, tedizolid, and radezolid to biofilms of Enterococcus faecalis. (A) The eradicated effect of Linezolid, tedizolid, and radezolid to established biofilms of Enterococcus faecalis. The $8 \mathrm{E}$. faecalis clinical isolates were allowed to form mature biofilms for $24 \mathrm{~h}$, following which the established biofilms were treated with Linezolid, tedizolid, and radezolid (at $8 \times$ their MICs for $48 \mathrm{~h}$ ). The remaining biofilm biomass was determined by crystal violet staining. The inhibited effect of sub-minimum

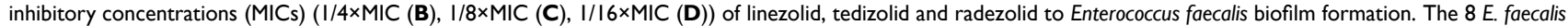
clinical isolates were treated with linezolid, tedizolid and radezolid at I/4xtheir MICs for $24 \mathrm{~h}$, and then biofilm biomass was determined by crystal violet staining. Data represent the average of three independent experiments (mean $\pm \mathrm{SD}$ ). $* P<0.05, * * P<0.0 \mathrm{I}, * * * P<0.00 \mathrm{I}$ (Student's $t$-test).

effect on the MICs of radezolid $(p<0.05)$. Further, the results of RT-qPCR showed that these antibiotics (linezolid, tedizolid and radzolide) does not influence the expression of efflux pump gene (OG1RF12220, OG1RF10126, and OG1RF10495) (data not shown).

\section{Discussion}

With the extensive growing use of antimicrobial agents, MDR Gram-positive strains have also grown over some of last year's. ${ }^{28}$ Linezolid has been extensively applied in clinical settings as a last-resort antimicrobial for treating the Gram-positive bacterial infections. ${ }^{33}$ However, with the increasing clinical use of linezolid, the clinical practice has observed appearance of linezolid-resistant strains. ${ }^{34}$ In this study, among the 2128 E. faecalis strains isolated from the First Affiliated Hospital of Wenzhou Medical University in 2011-2019, 3.34\% (71/2128) of E. faecalis were resistant to linezolid. Although our study showed that during a long period from 2011 to 2019, linezolid exhibited promising antimicrobial activity against clinically isolated E. faecalis, the resistance rate was low despite the emergence of the resistant strains. However, the emergence of resistant strains still was a great challenge, the new generation of oxazolidinone antibiotics development and application are urgent required. Our study further tested the antimicrobial activity of new oxazolidinone antibiotics tedizolid, and radezolid, against linezolidresistant clinical E. faecalis isolates. The results showed that the $\mathrm{MIC}_{50} / \mathrm{MIC}_{90}$ of tedizolid and radezolid exhibited 4- and 8- fold lower than linezolid, respectively. Although tedizolid and radezolid are the new generations of oxazolidinone antibiotics, the antibacterial activity showed a different effect compared with linezolid. The results suggested that the in vitro activity of radezolid against 
71 linezolid-resistant isolates was higher than that of tedizolid and linezolid. The differences between these three antibiotics are also worth further investigation in other aspects.

It is worth noting that the results of the gene carrying and mutation of oxazolidinone antimicrobial-resistant genes showed that 71 linezolid-resistant E. faecalis mainly had transferable gene $c f r$, $c f r(D)$, or optrA, and most strains carry optrA. This is consistent with previous reports. Thus, OptrA protein may play a significant role in mediating lowlevel linezolid resistance in E. faecalis. ${ }^{35}$ This gene codes for an ATP-binding cassette (ABC) transporter that confers resistance to oxazolidinones. ${ }^{36}$ Furthermore, $c f r$ and its variant $c f r(D)$ also decrease sensitivity to oxazolidinones. ${ }^{37}$ Therefore, the presence of optrA and $c f r$ is a concern because it may mediate multi-drug resistance. Of note, conjugation experiments with E. faecalis $\mathrm{JH} 2-2$ as recipients identified transferability of optrA gene between E. faecalis, which is consistent with the previous reports. Furthermore, there have been reports of easy transfer of optrA-carrying plasmids at high transfer frequencies of $10^{-2}-10^{-6}$ in vitro between human- and pig-derived E. faecalis ${ }^{38}$ Although, in our study, $17.91 \%$ (12/67) optrA confers transferable resistance to oxazolidinones, ${ }^{39}$ the presence of optrA may be in the plasmid or the chromosome in clinical isolates, which explain why the optrA in some strains cannot transfer. ${ }^{40}$ Additionally, none of the linezolid-resistant isolates had mutation in L3, L4, or L22 ribosomal proteins which is associated with the prolonged courses of linezolid administration and also an important mutation mechanism for growing linezolid resistance. ${ }^{41}$

A biofilm is a structured community of microorganisms adhering to a surface and exhibiting recalcitrance to antimicrobial compounds and resisting human immune defenses' action. ${ }^{42}$ The production of Enterococcus biofilms is widespread in Enterococcus faecalis, and the worldwide incidence rate is $26-100 \%{ }^{43}$ Studies have indicated that linezolid could affect the biofilms of E. faecalis. $^{44}$ Our research found that tedizolid had a significantly more significant inhibited effect on the biofilm formation of E. faecalis, which is higher than that of linezolid and radezolid. We further investigated whether this phenotypic was consistent with the eradication of established biofilms. Interestingly, we found that radezolid (at $8 \times$ their MIC) had the best scavenging effect on the established biofilms. Overall, the results show that the studied drugs in the biofilm inhibition have a significant difference, we speculated that the structure and impact of different drugs made specific differences in their biofilm activity. On the other hand, biofilm formation was regulated by biofilm-regulated genes (gelE, ace, esp). ${ }^{45}$ Different drugs may have different downregulation abilities on these genes. In short, comparing different antimicrobial biofilm inhibition, exploring related to clinical biofilm formation strain of clear, and the treatment of skin tissue infections have a particular guiding value. More experiments and studies are needed to investigate in the future.

Oxazolidinone antibacterial agents, such as linezolid, show promising effects on Gram-positive bacteria, but show poor activity on Gram-negative bacteria such as E. coli, mainly due to the RND-type efflux pump AcrB has the intense action. ${ }^{46}$ However, whether the efflux pump plays a role in the mechanism of Gram-positive bacteria resistant to linezolid, tedizolid or radezolid remains uncertain. In this study, the results indicated that among the three antimicrobial agents, the MICs of radezolid decreased most obviously after adding the addition of efflux pump inhibitor CCCP, followed by tedizolid. The efflux pump may be involved in the resistance to tedizolid and radezolid for E. faecalis isolates. However, we analyzed the effects of linezolid, tedizolid, and radzolide on the expression of efflux pump gene (OG1RF12220, $O G 1 R F 10126$, and $O G 1 R F 1049$ ) referring to Zheng et al, ${ }^{26}$ our results showed it did not influence these efflux pumps. The role of other efflux pump would also be studied in our future studies. The application of transcriptome sequencing will also be necessary to determine which efflux pump gene is active and to elucidate the specific mechanism in the future studies. In a word, the differences in the effect of efflux pumps of tedizolid, radezolid, and linezolid against linezolid-resistant isolates still need to be further investigated in the future.

In conclusion, the majority of E. faecalis isolates in our study were highly susceptible to linezolid. At the same time, both tedizolid and radezolid, the new generation of antimicrobial agents, showed different antibacterial activity to linezolid-resistant $E$. faecalis. The in vitro activity of radezolid against 71 linezolid-resistant isolates was higher than that of tedizolid and linezolid. Transferable genes $c f r, c f r(D)$, and optr $A$ may be involved in developing antimicrobial resistance to oxazolidinones of E. faecalis in this study. We also showed that radezolid was more effective in eradication biofilm, and tedizolid showed more effectiveness in inhibiting biofilm formation. Additionally, the efflux pump inhibition test results 
showed that the effluent pump had the most significant effect on the MICs of radezolid, but it is still essential to explore the mechanism of further study in the future. In a word, comparing the anti-microbic and anti-biofilm activity of three different oxazolidinone antibiotics can better understand them and guide clinical drug use may provide guiding value for clinical medication and treatment.

\section{Ethical Approval}

Whole investigation protocols in this study were approved by The Ethics Committee of the First Affiliated Hospital of Wenzhou Medical University.

\section{Acknowledgments}

We sincerely thank all the medical workers of the Department of Clinical Laboratory, the First Affiliated Hospital of Wenzhou Medical University for cooperating this study.

\section{Author Contributions}

All authors contributed to data analysis, drafting or revising the article, gave final approval of the version to be published, agreed to the submitted journal, and agreed to be accountable for all aspects of the work.

\section{Disclosure}

Lingbo Wang and Ying Zhang are co-first authors for this study. The authors report no conflicts of interest in this work.

\section{References}

1. Fiore E, Van Tyne D, Gilmore MS. Pathogenicity of enterococci. Microbiol Spectr. 2019;7. doi:10.1128/microbiolspec.GPP3-0053-2018

2. Kline KA, Lewis AL. Gram-positive uropathogens, polymicrobial urinary tract infection, and the emerging microbiota of the urinary tract. Microbiol Spectr. 2016;4. doi:10.1128/microbiolspec.UTI-00122012

3. Beganovic M, Luther MK, Rice LB, et al. A review of combination antimicrobial therapy for Enterococcus faecalis bloodstream infections and infective endocarditis. Clin Infect Dis. 2018;67:303-309. doi:10.1093/cid/ciy064

4. Gilmore MS, Salamzade R, Selleck E, et al. Genes contributing to the unique biology and intrinsic antibiotic resistance of Enterococcus faecalis. mBio. 2020;11. doi:10.1128/mBio.02962-20

5. Zurenko GE, Yagi BH, Schaadt RD, et al. In vitro activities of U-100592 and U-100766, novel oxazolidinone antibacterial agents. Antimicrob Agents Chemother. 1996;40:839-845. doi:10.1128/ AAC.40.4.839

6. Ippolito JA, Kanyo ZF, Wang D, et al. Crystal structure of the oxazolidinone antibiotic linezolid bound to the $50 \mathrm{~S}$ ribosomal subunit. J Med Chem. 2008;51:3353-3356. doi:10.1021/jm800379d
7. Aoki H, Ke L, Poppe SM, et al. Oxazolidinone antibiotics target the P site on Escherichia coli ribosomes. Antimicrob Agents Chemother. 2002;46:1080-1085. doi:10.1128/aac.46.4.1080-1085.2002

8. Wang J, Xia L, Wang R, et al. Linezolid and its immunomodulatory effect: in vitro and in vivo evidence. Front Pharmacol. 2019;10:1389. doi:10.3389/fphar.2019.01389

9. Liu BG, Yuan XL, He DD, et al. Research progress on the oxazolidinone drug linezolid resistance. Eur Rev Med Pharmacol Sci. 2020;24:9274-9281. doi:10.26355/eurrev_202009_23009

10. Burdette SD, Trotman R. Tedizolid: the first once-daily oxazolidinone class antibiotic. Clin Infect Dis. 2015;61:1315-1321. doi:10.1093/cid/civ501

11. Shaw KJ, Poppe S, Schaadt R, et al. In vitro activity of TR-700, the antibacterial moiety of the prodrug TR-701, against linezolid-resistant strains. Antimicrob Agents Chemother. 2008;52:4442-4447. doi:10.1128/AAC.00859-08

12. Jiang J, Hou Y, Duan M, et al. Design, synthesis and antibacterial evaluation of novel oxazolidinone derivatives nitrogen-containing fused heterocyclic moiety. Bioorg Med Chem Lett. 2021;32:127660. doi:10.1016/j.bmcl.2020.127660

13. Bassetti M, Righi E. Safety profiles of old and new antimicrobials for the treatment of MRSA infections. Expert Opin Drug Saf. 2016;15:467-481. doi:10.1517/14740338.2016.1142528

14. Carvalhaes CG, Sader HS, Flamm RK, et al. Assessment of tedizolid in vitro activity and resistance mechanisms against a collection of enterococcus spp. causing invasive infections, including isolates requiring an optimized dosing strategy for daptomycin from U.S. and European Medical Centers, 2016 to 2018. Antimicrob Agents Chemother. 2020;64. doi:10.1128/ AAC.00175-20

15. Torres C, Alonso CA, Ruiz-Ripa L, et al. Antimicrobial resistance in Enterococcus spp. of animal origin. Microbiol Spectr. 2018;6. doi:10.1128/microbiolspec.ARBA-0032-2018

16. Deshpande LM, Castanheira M, Flamm RK, et al. Evolving oxazolidinone resistance mechanisms in a worldwide collection of enterococcal clinical isolates: results from the SENTRY Antimicrobial Surveillance Program. $J$ Antimicrob Chemother. 2018;73:2314-2322. doi:10.1093/jac/dky188

17. Moure Z, Lara N, Marin M, et al. Interregional spread in Spain of linezolid-resistant Enterococcus spp. isolates carrying the optrA and poxtA genes. Int J Antimicrob Agents. 2020;55:105977. doi:10.1016/ j.ijantimicag.2020.105977

18. Li P, Yang Y, Ding L, et al. Molecular investigations of linezolid resistance in enterococci OptrA variants from a hospital in Shanghai. Infect Drug Resist. 2020;13:2711-2716. doi:10.2147/ IDR.S251490

19. Miller WR, Munita JM, Arias CA. Mechanisms of antibiotic resistance in enterococci. Expert Rev Anti Infect Ther. 2014;12:1221-1236. doi:10.1586/14787210.2014.956092

20. Klupp EM, Both A, Belmar Campos C, et al. Tedizolid susceptibility in linezolid- and vancomycin-resistant Enterococcus faecium isolates. Eur J Clin Microbiol Infect Dis. 2016;35:1957-1961. doi:10.1007/ s10096-016-2747-0

21. Bai B, Hu K, Li H, et al. Effect of tedizolid on clinical Enterococcus isolates: in vitro activity, distribution of virulence factor, resistance genes and multilocus sequence typing. FEMS Microbiol Lett. 2018;365. doi:10.1093/femsle/fnx284

22. Xu Z, Wei Y, Wang Y, et al. In vitro activity of radezolid against Enterococcus faecium and compared with linezolid. J Antibiot (Tokyo). 2020;73:845-851. doi:10.1038/s41429-020-0345-y

23. Lewis K. Riddle of biofilm resistance. Antimicrob Agents Chemother. 2001;45:999-1007. doi:10.1128/AAC.45.4.999-1007.2001

24. Bayston R, Ullas G, Ashraf W. Action of linezolid or vancomycin on biofilms in ventriculoperitoneal shunts in vitro. Antimicrob Agents Chemother. 2012;56:2842-2845. doi:10.1128/AAC.06326-11 
25. Holmberg A, Morgelin M, Rasmussen M. Effectiveness of ciprofloxacin or linezolid in combination with rifampicin against Enterococcus faecalis in biofilms. J Antimicrob Chemother. 2012;67:433-439. doi:10.1093/jac/dkr477

26. Zheng J, Chen Z, Lin Z, et al. Radezolid is more effective than linezolid against planktonic cells and inhibits Enterococcus faecalis Biofilm Formation. Front Microbiol. 2020;11:196. doi:10.3389/ fmicb.2020.00196

27. Performance CLSI. Standards for Antimicrobial Susceptibility Testing. 30th ed. CLSI supplement M100. Wayne, PA: Clinical and Laboratory Standards Institute; 2020.

28. Patel SN, Memari N, Shahinas D, et al. Linezolid resistance in Enterococcus faecium isolated in Ontario, Canada. Diagn Microbiol Infect Dis. 2013;77:350-353. doi:10.1016/j.diagmicrobio.20 13.08 .012

29. Ruiz-Ripa L, Fessler AT, Hanke D, et al. Mechanisms of linezolid resistance among enterococci of clinical origin in Spain-detection of optrA- and cfr(D)-carrying E. faecalis. Microorganisms. 2020;8:1155. doi:10.3390/microorganisms 8081155

30. Huys G, D'Haene K, Collard JM, et al. Prevalence and molecular characterization of tetracycline resistance in Enterococcus isolates from food. Appl Environ Microbiol. 2004;70:1555-1562. doi:10.1128/aem.70.3.1555-1562.2004

31. Zheng JX, Sun X, Lin ZW, et al. In vitro activities of daptomycin combined with fosfomycin or rifampin on planktonic and adherent linezolid-resistant isolates of Enterococcus faecalis. J Med Microbiol. 2019;68:493-502. doi:10.1099/jmm.0.000945

32. Mlynek KD, Callahan MT, Shimkevitch AV, et al. Effects of low-dose amoxicillin on Staphylococcus aureus USA300 Biofilms. Antimicrob Agents Chemother. 2016;60:2639-2651. doi:10.1128/ AAC.02070-15

33. Koulenti D, Xu E, Song A, et al. Emerging treatment options for infections by multidrug-resistant gram-positive microorganisms. Microorganisms. 2020;8:191. doi:10.3390/microorganisms8020191

34. Bai B, Hu K, Zeng J, et al. Linezolid consumption facilitates the development of linezolid resistance in Enterococcus faecalis in a tertiary-care hospital: a 5-Year Surveillance Study. Microb Drug Resist. 2019;25:791-798. doi:10.1089/mdr.2018.0005

35. Yan J, Xia Y, Yang M, et al. Quantitative proteomics analysis of membrane proteins in Enterococcus faecalis with low-level linezolid-resistance. Front Microbiol. 2018;9:1698. doi:10.3389/ fmicb.2018.01698

36. Cai J, Wang Y, Schwarz S, et al. Enterococcal isolates carrying the novel oxazolidinone resistance gene optrA from hospitals in Zhejiang, Guangdong, and Henan, China, 2010-2014. Clin Microbiol Infect. 2015;21:1095e1-4. doi:10.1016/j.cmi.2015.08.007
37. Guerin F, Sassi M, Dejoies L, et al. Molecular and functional analysis of the novel cfr(D) linezolid resistance gene identified in Enterococcus faecium. $J$ Antimicrob Chemother. 2020;75:1699-1703. doi:10.1093/jac/dkaa125

38. Shang Y, Li D, Shan X, et al. Analysis of two pheromone-responsive conjugative multiresistance plasmids carrying the novel mobile optrA locus from Enterococcus faecalis. Infect Drug Resist. 2019;12:2355-2362. doi:10.2147/IDR.S206295

39. Wang Y, Lv Y, Cai J, et al. A novel gene, optrA, that confers transferable resistance to oxazolidinones and phenicols and its presence in Enterococcus faecalis and Enterococcus faecium of human and animal origin. J Antimicrob Chemother. 2015;70:2182-2190. doi: $10.1093 / \mathrm{jac} / \mathrm{dkv} 116$

40. Zou J, Tang Z, Yan J, et al. Dissemination of linezolid resistance through sex pheromone plasmid transfer in Enterococcus faecalis. Front Microbiol. 2020;11:1185. doi:10.3389/fmicb.2020.01185

41. Gonzales RD, Schreckenberger PC, Graham MB, et al. Infections due to vancomycin-resistant Enterococcus faecium resistant to linezolid. Lancet. 2001;357:1179. doi:10.1016/S0140-6736(00)04376-2

42. Nostro A, Roccaro AS, Bisignano G, et al. Effects of oregano, carvacrol and thymol on Staphylococcus aureus and Staphylococcus epidermidis biofilms. $J$ Med Microbiol. 2007;56:519-523. doi:10.1099/jmm.0.46804-0

43. Tong $\mathrm{J}$, Jiang $\mathrm{Y}, \mathrm{Xu} \mathrm{H}$, et al. In vitro antimicrobial activity of fosfomycin, rifampin, vancomycin, daptomycin alone and in combination against vancomycin-resistant enterococci. Drug Des Devel Ther. 2021;15:3049-3055. doi:10.2147/DDDT.S315061

44. Luther MK, Arvanitis M, Mylonakis E, et al. Activity of daptomycin or linezolid in combination with rifampin or gentamicin against biofilm-forming Enterococcus faecalis or E. faecium in an in vitro pharmacodynamic model using simulated endocardial vegetations and an in vivo survival assay using Galleria mellonella larvae. Antimicrob Agents Chemother. 2014;58:4612-4620. doi:10.1128/ AAC.02790-13

45. Xiao B, Zou Z, Bhandari J, et al. Exposure to diode laser (810nm) affects the bacterial adherence and biofilm formation in a E. faecalis biofilm model. Photodiagnosis Photodyn Ther. 2020;31:101772. doi:10.1016/j.pdpdt.2020.101772

46. Schumacher A, Trittler R, Bohnert JA, et al. Intracellular accumulation of linezolid in Escherichia coli, Citrobacter freundii and Enterobacter aerogenes: role of enhanced efflux pump activity and inactivation. $J$ Antimicrob Chemother. 2007;59:1261-1264. doi:10.1093/jac/dk1380
Infection and Drug Resistance

\section{Publish your work in this journal}

Infection and Drug Resistance is an international, peer-reviewed openaccess journal that focuses on the optimal treatment of infection (bacterial, fungal and viral) and the development and institution of preventive strategies to minimize the development and spread of resistance. The journal is specifically concerned with the epidemiology of antibiotic resistance and the mechanisms of resistance development and diffusion in both hospitals and the community. The manuscript management system is completely online and includes a very quick and fair peerreview system, which is all easy to use. Visit http://www.dovepress.com/ testimonials.php to read real quotes from published authors. 